\title{
Optimal storage dispatch in a consumer setting with local generation resources
}

\author{
Dennis Metz \\ PDSSE Program, FEUP/MIT PhD Program \\ Faculty of Engineering, University of Porto \\ Rua Dr. Roberto Frias, 4200 - 465 Porto \\ Porto, Portugal \\ dennis.metz@fe.up.pt
}

\author{
João Tomé Saraiva \\ INESC TEC / DEEC \\ Faculty of Engineering, University of Porto \\ Rua Dr. Roberto Frias, 4200 - 465 Porto \\ Porto, Portugal \\ jsaraiva@fe.up.pt
}

\begin{abstract}
While in the past the installation of distributed generation systems was oftentimes motivated by attractive feedin tariffs, many consumers now install such systems to increase their self-sufficiency in order to avoid rising electricity cost. However, due to the intermittency of photovoltaic systems and the dependency of cogeneration units on the thermal demand, there remains a significant mismatch between local generation and demand. Electrical storage devices can align generation and demand, minimizing the mentioned gap and hence the amount of energy that needs to be taken from the grid. This paper describes a model to determine the optimal dispatch of such a system, taking the thermal system into account in order to account for the dependency of cogeneration units. A mixed integer program is presented, which identifies the cost minimizing operation schedule. This model is tested using a case study considering realistic values taken from the German case.
\end{abstract}

Index Terms - Energy storage, Power generation dispatch

\section{INTRODUCTION}

Over the last decade, distributed generation technologies led by photovoltaic (PV) systems have seen a strong growth in interest. In the early years of that period many photovoltaic systems were installed in Germany motivated by an attractive feed-in scheme for surplus generation. The wide-scale rollout of systems has led to decreasing technology cost, both due to technical advances as well as due to scale-effects in their production. However, while the strong growth in implementations is considered as an important step towards a more environmental friendly generation mix, it has also led to rising electricity cost for consumers, as the cost for the feedin tariffs is redistributed by means of a surcharge included in the electricity tariffs. Furthermore, as a reaction to decreasing technology cost, feed-in tariffs have been reduced over time. Therefore, the primary objective of most new installations has shifted towards substituting energy taken from the grid by local generation.

However, the self-consumption is limited by the availability of the intermittent and hence non-dispatchable photovoltaic generation. Weniger et al. [1] and CastilloCagigal et al. [2] determined a self-sufficiency rate for residential consumers of about $30 \%$. Demand-side management allows consumers to further increase the share of generated energy consumed locally. However, this comes typically at the cost of reduced comfort at time. Furthermore, the extent of demand that can be aligned to generation is limited, such that some residual demand always remains. Castillo-Cagigal et al. [2] found that up to $57 \%$ of local demand can be supplied by photovoltaic generation.

On the other hand, storage devices allow consumers to shift locally generated energy in time without changing their consumption pattern. Surplus generation, that is the local generation that exceeds instantaneous demand, is stored instead of being fed into the grid. During times, when demand exceeds local generation, the stored energy can then be locally consumed. A storage device therefore allows consumers to reduce the amount of energy taken from the grid and maximize the usage of locally generated energy.

However, the integration of a storage device raises the following question - how much grid demand can be substituted by energy from local generation resources. This problem has been previously considered by several authors. Bost et al. [3] find that installing a storage system at a typical household increases the local consumption from $20-40 \%$ to $40-96 \%$. The magnitude of these numbers is confirmed by Weniger et al. [1], who found an increase from around $30 \%$ to about $60 \%$. Castillo-Cagigal et al. [2] determined a selfsufficiency of about $70 \%$ and concluded that the benefits of demand-side management diminish with increasing storage capacity and become insignificant when the storage capacity exceeds daily demand. In a comprehensive review about the potential of pursuing demand-side management or installing a storage device, Luthander et al. [4] found that storage devices can typically increase self-consumption by $13-24 \%$, whereas demand-side management is less effective and leads only to increases of $2 \%-15 \%$.

Hawkes and Leach [5] analyzed the cost-minimizing dispatch for cogeneration units. They found that the least cost is achieved when the unit is dispatched against both heat and electric demand. Furthermore, they identified differences between winter and summer due to the different overall thermal load. Barbieri et al. [6] found that a cogeneration unit can supply up to $85 \%$ of local electric demand. Ren and Gao [7] report values between $24 \%$ and $69 \%$, depending on the chosen technology as well as the system capacity. 
The previous work has shown that storage can significantly increase the usage of locally generated energy. However, the analyses were restricted to generation from photovoltaic systems. The work now being reported considers in addition cogeneration units (CHP) as a second major distributed generation technology. Cogeneration units use the waste heat from the power generation process to satisfy local heat demand and hence are able to use the consumed fuel more efficiently. They are therefore an interesting option for consumers, who require both heat as well as electricity. Contrary to photovoltaic systems, cogeneration units are dispatchable and can react to the varying electric demand. Nonetheless, their operational flexibility is impacted by the interaction and requirements of the thermal system. Furthermore, feed-in of surplus generation from cogeneration units is even less attractive than for photovoltaic systems. Therefore, the deployment of storage in combination with a cogeneration unit can be valuable.

It should be noted that the objective of this work is the development of a dispatch model, which minimizes the operating cost. Therefore, on purpose, no investment cost was considered at this stage. The result therefore will be the cost minimizing dispatch for a given system configuration without any statement about the optimality of the configuration.

\section{DEVELOPED APPROACH}

To determine the optimum operation of storage in such a co-integrated system, both the electric as well as the thermal system and the respective energy flows must be considered in the problem formulation, which significantly increases the complexity of the problem. In order to model the thermal system, a range of additional system components besides the cogeneration unit will be considered: a gas boiler, solar thermal generation and heat-pumps, which represent an additional link between the two systems by converting electric to thermal energy. Furthermore, contrary to the electric system where power can be exchanged with the grid and hence shortfalls or surplus from local generation can be easily balanced, all thermal demand must effectively be satisfied by local generation. Therefore, thermal storage will be considered as well.

Power flows will be denoted by $P(t)$, heat flows by $Q(t)$. Local power demand $\left(P_{\text {Load }}\right)$ as well as the power demand from the heat pump $\left(P_{H P}\right)$ are primarily satisfied by the generation from a photovoltaic system $\left(P_{P V}\right)$ and a cogeneration unit $\left(P_{C H P}\right)$. Shortfalls or surplus generation can be balanced with the storage device $\left(P_{\text {ElStorage }}\right)$ and exchanged with the grid $\left(P_{G r i d}\right)$. Demand for heat $\left(Q_{\text {Load }}\right)$ is satisfied by solar thermal $\left(Q_{S T}\right)$, gas boiler $\left(Q_{G B}\right)$, heat pump $\left(Q_{H P}\right)$ and the cogeneration unit $\left(Q_{C H P}\right)$. A thermal storage device $\left(Q_{\text {ThStorage }}\right)$ is utilized to balance residuals. Figure 1 shows the considered energy flows as well as the interconnection between the heat and the power systems, represented by the cogeneration unit as well as the heat pump.

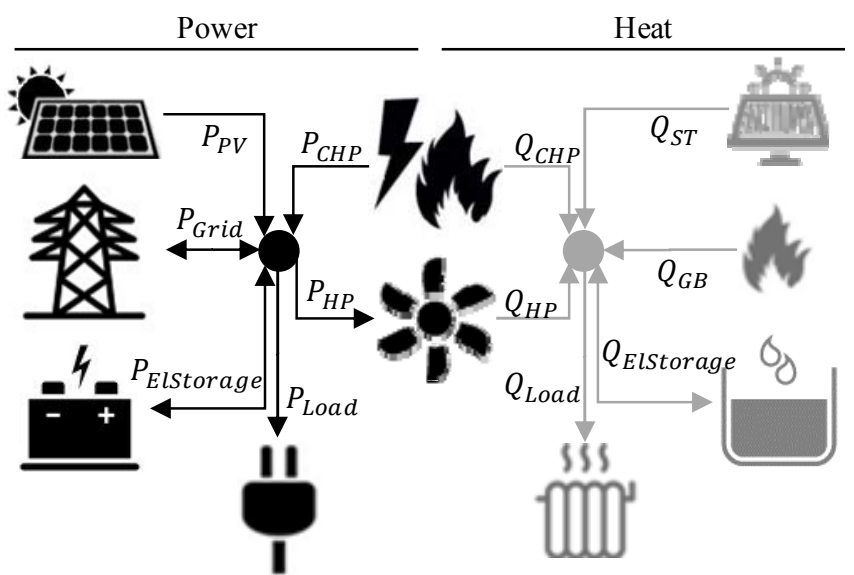

Figure 1: Simplified representation of power and heat flows.

In order to determine the cost minimizing operation schedule, a mixed integer program (MIP) was formulated. This problem optimizes an outcome described and constrained by linear relationships. These represent the functionality of the considered system in an abstracted model. However, contrary to a linear problem, some of the variables can only assume integer values. The MIP is described by equations (1) - (22). The optimization horizon of the problem is $T$. Each time step is referred to by its index $t$ and has a duration $\Delta t$, expressed as fraction or multiple of an hour. All variables are assumed to be constant during each time step. Positive values for $P(t)$ and $Q(t)$ represent a source of energy, whereas negative values reflect a demand for energy.

Table 1 summarizes the relevant system parameters and Table 2 lists the simulation variables. Subscript of parameters refer to the related system component, and superscripts distinguish additional attributes.

TABLE 1: SYSTEM PARAMETERS

\begin{tabular}{|c|c|c|}
\hline$E_{\text {ElStorage }}^{\text {Capacity }}$ & [Wh] & Nominal capacity \\
\hline $\begin{array}{l}P_{\text {Elstorage/CHP/PV/HP }}^{\text {Capacity }} \\
Q_{\text {ThStorage / CHP/GB / ST }}^{\text {Capacity }}\end{array}$ & {$[\mathrm{W}]$} & Power / thermal capacities \\
\hline$P_{P V}^{\max f e e d-i n}$ & [W] & $\begin{array}{l}\text { Maximum permissible feed-in } \\
\text { power }\end{array}$ \\
\hline$P_{C H P}^{\text {Capacity }}$ & {$[\mathrm{W}]$} & Minimum power output \\
\hline$\delta_{\text {ElStorage }}$ & {$[\%]$} & Depth of discharge \\
\hline$\eta_{\text {ElStorage }}^{\text {In / Out }}$ & {$[\%]$} & $\begin{array}{l}\text { Charging / discharging } \\
\text { efficiency }\end{array}$ \\
\hline$\Phi_{\text {ThStorage }}$ & {$[\%]$} & Self-discharge per hour \\
\hline$R_{\text {Grid }}^{\text {Import }}(t)$ & {$[€ / \mathrm{Wh}]$} & Electricity tariff \\
\hline$R_{P V / C H P}^{\text {Export }}(t)$ & {$[€ / \mathrm{Wh}]$} & Feed-in tariffs \\
\hline$C_{C H P / G B}^{\text {Variable }}(t)$ & {$[€ / \mathrm{Wh}]$} & Operating cost of system \\
\hline $\operatorname{CoP}(t)$ & - & $\begin{array}{l}\text { Coefficient of performance of } \\
\text { a heat pump }\end{array}$ \\
\hline
\end{tabular}


TABLE $2 \cdot$ SimUlation VARIABLES

\begin{tabular}{|c|c|c|}
\hline$E_{\text {Storage }}(t)$ & {$[\mathrm{Wh}]$} & Current charge of the storage \\
\hline$Q_{L o a d / C H P / G B / S T / H P}(t)$ & {$[\mathrm{W}]$} & Heat flow \\
\hline$Q_{\text {ThStorage }}(t)$ & [W] & $\begin{array}{l}\text { Heat absorbed / supplied by the } \\
\text { thermal storage device }\end{array}$ \\
\hline$P_{\text {Load } / C H P / P V / H P}(t)$ & {$[\mathrm{W}]$} & Power flow \\
\hline $\begin{array}{l}P_{\text {Grid }}^{\text {Import }}(t) \\
P_{\text {Grid }}^{\text {Export } P V}(t) \\
P_{\text {Grid }}^{\text {Export CHP }}(t)\end{array}$ & {$[\mathrm{W}]$} & Power exchange with the grid \\
\hline $\begin{array}{l}P_{P V}^{*}(t) \\
Q_{S T}^{*}(t)\end{array}$ & [W] & $\begin{array}{l}\text { Available generation from a PV / } \\
\text { ST system }\end{array}$ \\
\hline $\begin{array}{l}P_{\text {ElStorage }}^{\text {In }}(t) \\
P_{\text {ElStorage }}^{\text {Out }}(t)\end{array}$ & [W] & $\begin{array}{l}\text { Power absorbed / supplied by the } \\
\text { electric storage device }\end{array}$ \\
\hline$y(t)$ & - & $\begin{array}{l}\text { Binary decision variable for the } \\
\text { operation of the storage device }\end{array}$ \\
\hline$z(t)$ & - & $\begin{array}{l}\text { Binary decision variable for the } \\
\text { operation of the CHP unit }\end{array}$ \\
\hline
\end{tabular}

The objective of the formulation (1) is the minimization of the operating cost, driven by the cost for the energy taken from the grid as well as the fuel cost for the operation of the cogeneration unit and the gas burner.

$\min \Delta t \times$

$\sum_{t=1}^{T}\left(\begin{array}{l}P_{\text {Grid }}^{\text {Import }}(t) \times R^{\text {Import }}(t)+P_{\text {Grid }}^{\text {Export } P V}(t) \times R_{P V}^{\text {Export }}(t)+ \\ P_{\text {Grid }}^{\text {Export CHP }}(t) \times R_{C H P}^{\text {Export }}(t)+Q_{G B}(t) \times C_{G B}^{\text {Variable }}+ \\ \left(P_{C H P}(t)+Q_{C H P}(t)\right) \times C_{C H P}^{\text {Variable }}\end{array}\right.$

In order to accurately reflect reality, the MIP is restricted by several limitations. First, equation (2) ensures the power balance, that is the constant equilibrium of supply and demand.

$$
\begin{aligned}
& P_{\text {Grid }}^{\text {Import }}(t)+P_{\text {Grid }}^{\text {Export } P V}(t)+P_{\text {Grid }}^{\text {Export CHP }}(t)+ \\
& P_{\text {ElStorage }}^{\text {In }}(t)+P_{\text {ElStorage }}^{\text {Out }}(t)+ \\
& P_{P V}(t)+P_{C H P}(t)+P_{H P}(t)+P_{\text {Load }}(t)=0
\end{aligned}
$$

The output of the cogeneration can either be zero or be modulated between a minimum and maximum output. Therefore, a binary decision variable $z(t)$ is introduced, which reflects the current state of operation. For $z(t)=0$, the cogeneration unit is switched off. It is assumed to be operating for $z(t)=1$. Equation (3) ensures that when the cogeneration unit is operating, at least the minimum output is generated. Equation (4) limits the output to the capacity.

$$
\begin{aligned}
& -z(t) \times P_{C H P}^{\text {CapacityMin }}+P_{C H P}(t) \geq 0 \\
& -z(t) \times P_{C H P}^{\text {Capacity }}+P_{C H P}(t) \leq 0
\end{aligned}
$$

The power considered of the photovoltaic system is limited to current generation as indicated by equation (5).

$0 \leq P_{P V}(t) \leq P_{P V}^{*}(t)$

To properly account for the energy exchange with the grid and the associated consumption and feed-in tariffs, the power exchange is broken into individual vectors. Equation (6) ensures that the power taken from the grid is a positive number.

$0 \leq P_{\text {Grid }}^{\text {Import }}(t)$

Furthermore, equations (7) and (8) limit the feed-in of power from the photovoltaic system and the cogeneration unit to their current generation.

$$
\begin{aligned}
& P_{C H P}(t)+P_{\text {Grid }}^{\text {ExportCHP }}(t) \geq 0 \\
& P_{P V}(t)+P_{\text {Grid }}^{\text {ExportPV }}(t) \geq 0
\end{aligned}
$$

Equation (9) ensures that feed-in does not exceed the feed-in limit for photovoltaic generation, which is for example a regulatory requirement in Germany.

$-P_{P V}^{\max \text { feed-in }} \leq P_{\text {Grid }}^{\text {ExportPV }}(t) \leq 0$

Equations (10) - (13) reflect the operation of the electrical storage device. To accurately incorporate the efficiency losses in the charge-/ discharge process, the process had to be broken into two separate vectors represented by $P_{\text {ElStorage }}^{I n}$ and $P_{\text {ElStorage }}^{\text {Out }}$. The binary variable $y(t)$ ensures that the storage device is either charging $(y(t)=1)$ or discharging $(y(t)=0)$. The associated power flows are limited to the power rating of the storage device by equations (10) and (11).

$$
\begin{aligned}
& 0 \leq P_{\text {ElStorage }}^{\text {Out }}(t) \leq P_{\text {ElStorage }}^{\text {Capacity }} \times(1-y(t)) \\
& -P_{\text {ElStorage }}^{\text {Capacity }} \times y(t) \leq P_{\text {ElStorage }}^{I n}(t) \leq 0
\end{aligned}
$$

The state of charge of the storage device is bound by its energy capacity as indicated by equations (12) and (13), 
considering charging- and discharging efficiencies $\eta_{\text {ElStorage }}^{\text {In }}$ and $\eta_{\text {ElStorage }}^{\text {Out }}$ as well as the depth of discharge $\delta_{\text {ElStorage. }}$

$$
\begin{gathered}
\sum_{n=1}^{t} P_{\text {ElStorage }}^{I n}(n) \times \eta_{\text {ElStorage }}^{\text {In }} \times \Delta t+ \\
\sum_{n=1}^{t} P_{\text {ElStorage }}^{\text {Out }}(n) \times 1 / \eta_{\text {ElStorage }}^{\text {Out }} \times \Delta t \leq 0 \\
\sum_{n=1}^{t} P_{\text {ElStorage }}^{I n}(n) \times \eta_{\text {ElStorage }}^{\text {In }} \times \Delta t+\quad \times \Delta t \\
\sum_{n=1}^{t} P_{\text {ElStorage }}^{\text {Out }}(n) \times 1 / \eta_{\text {ElStorage }}^{\text {Out }} \times \\
\geq-E_{\text {ElStorage }}^{\text {Capacity }} \times\left(1-\delta_{\text {ElStorage }}\right)
\end{gathered}
$$

Analogous to equation (2), also the thermal system must be balanced as indicated by equation (14).

$$
\begin{gathered}
Q_{C H P}(t)+Q_{S T}(t)+Q_{G B}(t)+Q_{H P}(t)+ \\
Q_{\text {ThStorage }}(t)+Q_{\text {Load }}(t)=0
\end{gathered}
$$

The heat output of the cogeneration unit $Q_{C H P}(t)$ is defined according to the chosen power output and the constant ratio of the system capacities as described by equation (15).

$Q_{C H P}(t)=P_{C H P}(t) \times \frac{Q_{C H P}^{\text {Capacity }}}{P_{C H P}^{\text {Capacity }}}$

Equation (16) limits the heat contribution of the solar thermal installation $Q_{S T}(t)$ to the current generation.

$$
0 \leq Q_{S T}(t) \leq Q_{S T}^{*}(t)
$$

The output of the gas boiler as well as of the heat pump is limited to its thermal rating, as indicated by equation (17).

$$
0 \leq Q_{G B}(t) \leq Q_{G B}^{\text {Capacity }}
$$

The thermal output of the heat pump is linked to the chosen power $P_{H P}(t)$ as well as the coefficient of performance $\operatorname{CoP}(t)$, as shown by equation (18).

$$
Q_{H P}(t)=-P_{H P}(t) \times C o P(t)
$$

Equation (19) limits the power consumption and therefore also the thermal output of the heat pump to its rated capacity.
$-P_{H P}^{\text {Capacity }} \leq P_{H P}(t) \leq 0$

As the efficiency of the thermal process is assumed to be $100 \%$, the charging- and discharging of the thermal storage does not need to be broken into two separate vectors. Equation (20) limits the heat flows of the thermal storage to its capacity.

$-Q_{\text {ThStorage }}^{\text {Capacity }} \leq Q_{\text {ThStorage }}(t) \leq Q_{\text {ThStorage }}^{\text {Capacity }}$

Taking the self-discharge $\phi_{\text {ThStorage }}$ into account, equations (21) and (22) ensure that the state of charge of the thermal storage device stays within the operational limits and is feasible.

$\sum_{n=1}^{t} Q_{\text {ThStorage }}(n) \times \Delta t \times\left(1-\Delta t \times \phi_{\text {ThStorage }}\right)^{t-n} \leq 0$

$\sum_{n=1}^{t} Q_{\text {ThStorage }}(n) \times \Delta t+\left(1-\Delta t \times \phi_{\text {ThStorage }}\right)^{t-n} \geq-Q_{\text {ThStorage }}^{\text {Capacity }}$

As the computational complexity of the MIP grows significantly for longer time horizons $T$ and becomes quickly unsolvable, the problem will be split into smaller, more manageable time periods. In order to consider the timeshifting capability of the storage device and optimize across periods, an overlapping period will be considered for each optimization. Due to the daily periodicity of electric and thermal demand as well as of the photovoltaic and solar thermal generation, an optimization horizon of 24 hours with an overlapping window of 6 hours was found to produce only insignificant deviations from the results provided by an optimization across the complete time horizon.

\section{CASE STUdy}

To verify the model and determine the saving potential from installing a storage device, a case study was implemented. Therefore, a multi-family house in Germany with an annual electric demand of $150 \mathrm{MWh}$ and a thermal demand of $220 \mathrm{MWh}$ is considered. A synthetic time series with $\Delta t=0,25 h$ and $T=35040 h$ (1 year) is provided by [8], solar radiation is based on historical data available in [9].

The energy taken from the grid is charged at $R_{\text {Grid }}^{\text {Import }}(t)=0,265 € / \mathrm{kWh}$, whereas the feed-in of surplus generation is compensated with $R_{C H P}^{\text {Export }}(t)=0,035 € / \mathrm{kWh}$ and $R_{P V}^{\text {Export }}(t)=0,12 € / k W h$.

The following system configuration is assumed:

- Photovoltaic system with $P_{P V}^{\text {Capacity }}=20 \mathrm{~kW}$. The feed-in is limited to $P_{P V}^{\max }$ feed-in $=14 \mathrm{~kW}$; 
- Co-generation unit with $P_{C H P}^{\text {Capacity }}=16 \mathrm{~kW}$ and $Q_{\text {CHP }}^{\text {Capacity }}=35,2 \mathrm{~kW}$. The output unit can be modulated down to $P_{C H P}^{\text {CapacityMin }}=6,4 \mathrm{~kW}$, where the heat output scales linearly with the power output. Cost of operation $C_{C H P}^{\text {Variable }}$ are assumed to be $0,07 € / \mathrm{kWh}$;

- Solar thermal system with $Q_{S T}^{\text {Capacity }}=20 \mathrm{~kW}$;

- Gas boiler with $Q_{G B}^{\text {Capacity }}=100 \mathrm{~kW}$ and $C_{G B}^{\text {Variable }}=0,05 € / \mathrm{kWh} ;$

- Heat-pump with $P_{H P}^{\text {Capacity }}=15 \mathrm{~kW}$. The thermal output depends on the coefficient of performance $\operatorname{CoP}(t)$, defined by a piecewise linear relationship and ranges from 2 to 4,7 .

Furthermore, the following storage devices are integrated:

- Electrical storage device with $E_{\text {ElStorage }}^{\text {Capacity }}=50 \mathrm{kWh}$, $P_{\text {ElStorage }}^{\text {Capacity }}=20 \mathrm{~kW}, \delta_{\text {ElStorage }}=25 \%$ and $\eta_{\text {ElStorage }}^{\text {In }} /$ $\eta_{\text {ElStorage }}^{\text {Out }}=95 \%$. Self-discharge is neglected;

- Thermal storage device with $E_{\text {ThStorage }}^{\text {Capacity }}=100 \mathrm{kWh}$ and $P_{\text {ThStorage }}^{\text {Capacity }}=50 \mathrm{~kW}$. The storage is assumed to self-discharge by $\phi_{\text {ThStorage }}=0,35 \%$ per hour. No efficiency losses or minimum depth of discharge are considered.

Further taxes, subsidies and fees are neglected for this analysis.

\section{RESULTS}

The formulation described by equations (1) - (22) was tested assuming the above-mentioned system configuration, spanning both the electric and thermal subsystems. Solving the MIP resulted in an annual operating cost of 32000 EUR. As a reference, setting $E_{\text {ElStorage }}^{\text {Capacity }}=0$ and solving the MIP again results in an annual cost of 32719 EUR. Hence, the annual savings in operating cost induced by the installation of a storage device therefore amount to 719 EUR.

Figure 2 shows the resulting heat flows for the optimal dispatch including the storage device. Most of the heat is generated by the cogeneration unit. In addition, the gas boiler is also frequently dispatched in order to cover peak heat demand. Interestingly, the theoretic annual generation from the solar thermal system of $20 \mathrm{MWh}$ is mostly not considered (as permitted by equation (16)). Apparently, the cost advantage of local generated electricity exceeds the benefits of free heat.
The contribution from the heat pump is neglectable. However, when no electrical storage device is present, the heat pump is dispatched more frequently (14 MWh) to absorb electrical surplus generation instead of feeding it into the grid. In total, about one third of heat generation is not immediately used, but stored temporarily. However, the thermal losses from self-discharge are low, indicating that the heat is stored only for rather short intervals.

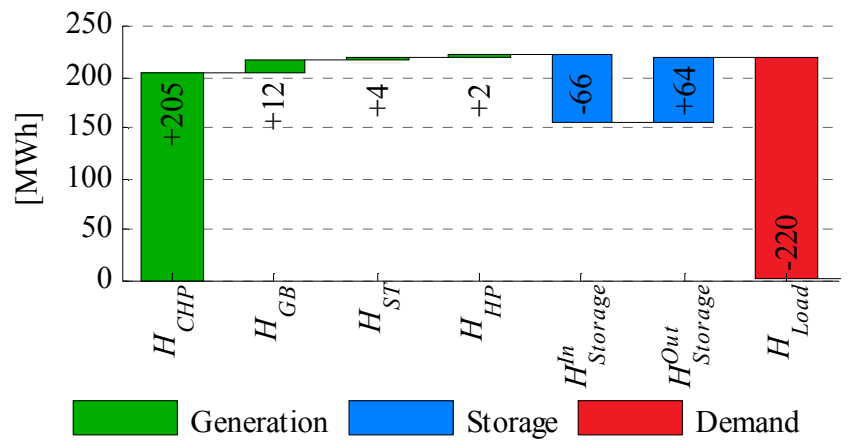

Figure 2: Heat flows.

The break-down of power flows is shown in Figure 3. Most of the demand is supplied by the cogeneration unit. The remainder was either balanced by the PV system or taken from the grid. Almost no energy was fed-in. In about 200 cycles, the electrical storage device shifted about 7.3 MWh in total. Without the storage device, $46 \mathrm{MWh}$ have to be taken from the grid.

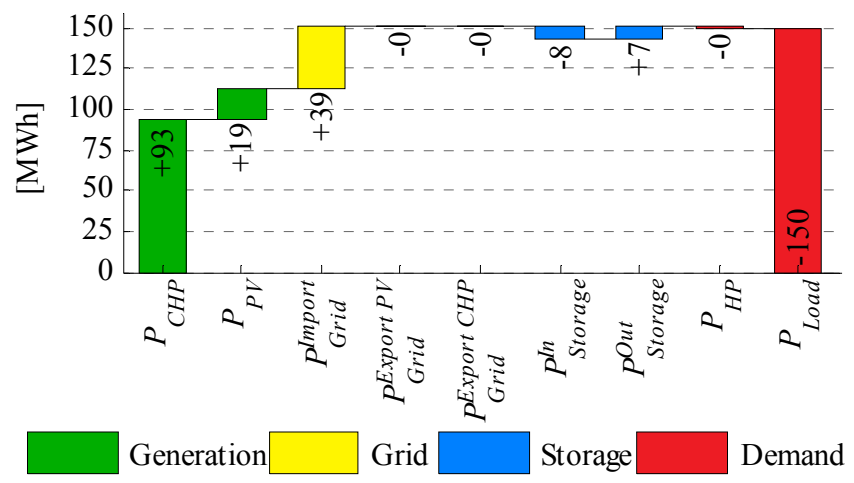

Figure 3: Power flows.

Figure 4 shows the benefit of adding an electrical storage device. The annual electricity bill is reduced by 1903 EUR, as significantly less energy $(-7 \mathrm{MWh})$ needs to be taken from the grid. On the other hand side, the cost for the operation of the CHP increases, as it is operating now for about 250 hours more. In addition, the cost for the gas boiler is also higher, as previous heat generation from the heat pump must be substituted. The feed-in of surplus generation is further reduced, resulting in slightly lower revenues. 


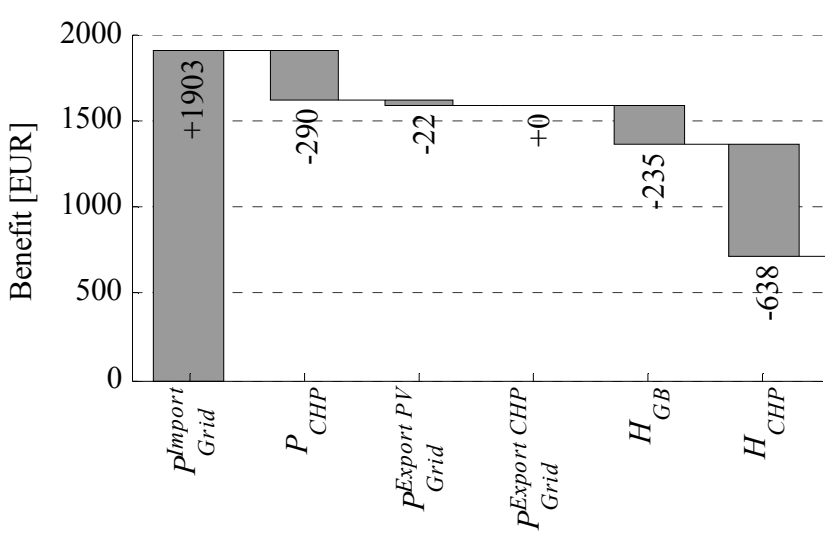

Figure 4: Benefit of installing the electrical storage system.

Last, Figure 5 shows the evolution of the state of charge of the electrical storage along the year. There is an obvious difference between summer and winter periods, driven by the demand for heat and the resulting dispatch of the cogeneration unit. During the winter, the electrical storage device typically provides energy around noon and especially in the evening hours. It is charged during the rest of the day from the surplus generation of the cogeneration unit. During the summer, the storage device remains idle most of the time. Hence, little surplus generation from the PV system is available, indicating that the capacity of the system could be further increased.

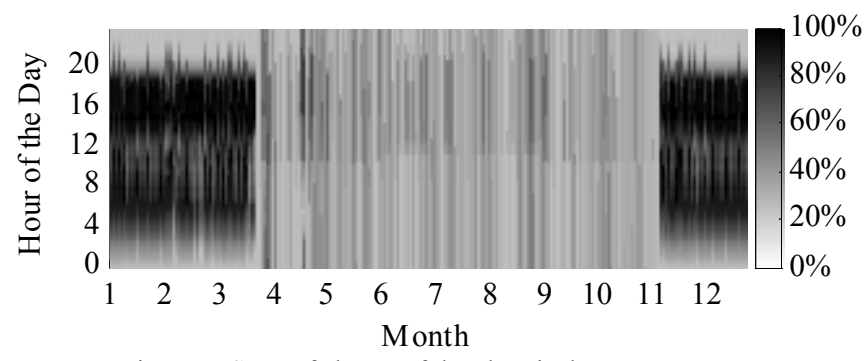

Figure 5: State of charge of the electrical storage system.

\section{CONCLUSIONS AND FUTURE WORK}

In this work, a mixed integer program was presented, which allows to determine the cost minimizing dispatch for a given system configuration. Besides the electrical system, the thermal system was taken into account in order to consider the dependency of the cogeneration unit. The approach was tested using historical data for an assumed system located in Germany.

It was found that electrical storage can reduce the operating cost of the global system. The cost for energy taken from the grid was reduced substantially. However, half of the benefit was erased again by the increased cost for the provision of heat. The feed-in of power was reduced to zero. Given the presence of a storage device, the self-sufficiency was increased from $69 \%$ to $74 \%$. At the same time, the self- consumption of local electric generation increased from $99.2 \%$ to $100 \%$.

The installation of an electrical storage device also affects the heat system, as generation exceeding instantaneous demand is now rather stored than used for the heat pump, which again results in a more frequent dispatch of the gas boiler. Furthermore, also the cogeneration unit is now operated more frequently. Surprisingly, a significant share of solar thermal generation was rejected. Instead, it was more beneficial to dispatch the cogeneration unit as the cost advantage of substituting energy taken from the grid with local generation exceeded the additional cost.

The focus of the current work was to optimize the dispatch of an existing installation with specified system capacities. However, the current approach does not consider investment cost. From the results it becomes obvious that both a heat pump as well as a solar thermal system are not dispatched frequently. Furthermore, while electrical storage clearly adds value, a complete analysis should also consider its investment cost. Hence, in a future work the designed approach to determine the cost minimizing dispatch should be embedded in a search routine to identify the optimal capacities of all the considered subsystems.

\section{ACKNOWLEDGMENT}

The first author would like to thank Fundação para a Ciência e Tecnologia, FCT, that partially funded this research work through the $\mathrm{PhD}$ grant no $\mathrm{PD} / \mathrm{BD} / 105848 / 2014$.

\section{REFERENCES}

[1] J. Weniger, T. Tjaden, and V. Quaschning, "Sizing of Residential PV Battery Systems", Energy Procedia, vol. 46, pp. 78-87, 2014.

[2] M. Castillo-Cagigal, E. Caamaño-Martín, E. Matallanas, D. MasaBote, A. Gutiérrez, F. Monasterio-Huelin, and J. Jiménez-Leube, "PV self-consumption optimization with storage and Active DSM for the residential sector", Sol. Energy, vol. 85, no. 9, pp. 23382348, Sep. 2011.

[3] M. Bost, B. Hirschl, and A. Aretz, "Effekte von Eigenverbrauch und Netzparität bei der Photovoltaik", Institut für ökologische Wirtschaftsforschung, Berlin, Germany, 2011.

[4] R. Luthander, J. Widén, D. Nilsson, and J. Palm, "Photovoltaic self-consumption in buildings: A review", Appl. Energy, vol. 142, pp. 80-94, 2015.

[5] A. D. Hawkes and M. A. Leach, "Cost-effective operating strategy for residential micro-combined heat and power", Energy, vol. 32, no. 5, pp. 711-723, 2007.

[6] E. S. Barbieri, P. R. Spina, and M. Venturini, "Analysis of innovative micro-CHP systems to meet household energy demands", in Energy Solutions for a Sustainable World Proceedings of the Third International Conference on Applied Energy, 2012, pp. 723-733.

[7] H. Ren and W. Gao, "Economic and environmental evaluation of micro CHP systems with different operating modes for residential buildings in Japan", Energy Build., vol. 42, no. 6, pp. 853-861, 2010.

[8] G. Dubielzig, Referenzlastprofile von Ein- und Mehrfamilienhäusern für den Einsatz von KWK-Anlagen. Düsseldorf: VDI Verlag, 2007.

[9] "SoDa - Free time-series of solar radiation data." [Online]. Available: http://www.sodais.com/eng/services/services_radiation_free_eng.php. 